Modern Physics Letters A

(C) World Scientific Publishing Company

\title{
REVIEW OF RECENT RESULTS FROM THE RHIC BEAM ENERGY SCAN
}

\author{
LOKESH KUMAR \\ School of Physical Sciences, National Institute of Science Education and Research, \\ Bhubaneswar, Odisha 751005, India \\ lokesh@rcf.rhic.bnl.gov; lokesh@niser.ac.in
}

\begin{abstract}
We review recent results from the RHIC beam energy scan (BES) program, aimed to study the Quantum Chromodynamics (QCD) phase diagram. The main goals are to search for the possible phase boundary, softening of equation of state or first order phase transition, and possible critical point. Phase-I of the BES program has recently concluded with data collection for $\mathrm{Au}+\mathrm{Au}$ collisions at center-of-mass energies $\left(\sqrt{s_{N N}}\right)$ of $7.7,11.5$, 19.6, 27, and $39 \mathrm{GeV}$. Several interesting results are observed for these lower energies where the net-baryon density is high at the mid-rapidity. These results indicate that the matter formed at lower energies $(7.7$ and $11.5 \mathrm{GeV})$ is hadron dominated and might not have undergone a phase transition. In addition, the centrality dependence of freeze-out parameters is observed for the first time at lower energies, slope of directed flow for (net)-protons measured versus rapidity shows an interesting behavior at lower energies, and higher moments of net-proton show deviation from Skellam expectations at lower energies. An outlook for the future BES Phase-II program is presented and efforts for the detailed study of QCD phase diagram are discussed.
\end{abstract}

Keywords: Quark Gluon Plasma; QCD phase diagram; QCD critical point; Phase transition; Chemical and Kinetic freeze-out; Directed and elliptic flow, dynamical charge correlations, Eccentricity, Nuclear modification factor.

PACS Nos.: 25.75.-q,25.75.Nq, 12.38.Mh,25.75.Dw,25.75.Gz,25.75.Ld

\section{Introduction}

The main goals of high-energy heavy-ion collision experiments are to search and study the hot and dense matter called Quark-Gluon Plasma (QGP) formed in these collisions 1. Moreover, there is a great interest in understanding the QCD phase diagram, a phase diagram for strong interactions, to the level of that for electromagnetic interactions such as water. The results from top RHIC energies suggest the formation of QGP 1 . The focus has now shifted to study the QGP properties 2 and establish the QCD phase diagram. In this review, we will concentrate on the latter part which is establishing the QCD phase diagram through a dedicated program at RHIC called beam energy scan program $314 \mid 5$. Figure 1 shows the schematic QCD phase diagram plotted as temperature $T$ vs. baryonic chemical potential $\mu_{B}$. 6 . There are two main phases predicted in the QCD phase diagram: QGP and hadronic 


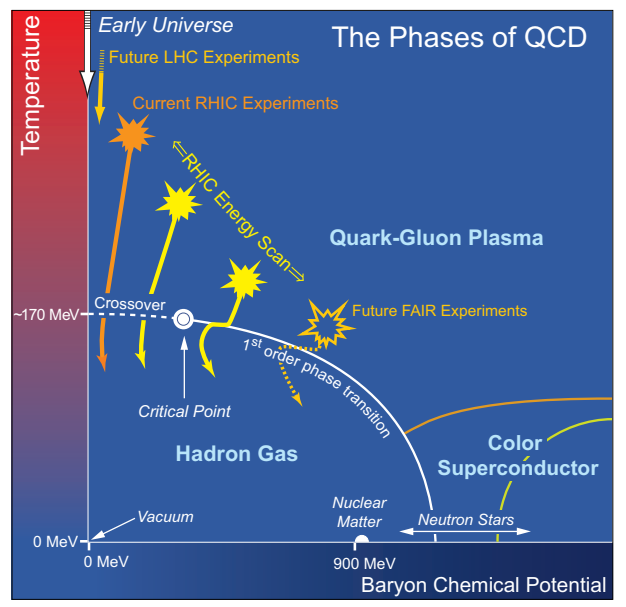

Fig. 1. (Color online) Schematic QCD phase diagram plotted as temperature $T$ versus baryon chemical potential $\mu_{B}$.

gas phase. Lattice QCD calculations predict that the transition between QGP and the hadronic gas at baryonic chemical potential $\mu_{B}=0$ is a crossover 7 . At large $\mu_{B}$, the transition between QGP and hadron gas is expected to be a first order phase transition 899 . Subsequently, the end-point of this first-order phase transition line (while going towards the crossover) would be the position of a critical point 10 . While there is a little guidance from the theory side about the QCD phase diagram, efforts are ongoing from the experimental side to establish some of its distinct structures such as phase boundary between de-confined phase of quarks and gluons and hadron gas phase, first-order phase transition line, and the critical point.

Experimentally, the two axes: $T$ and $\mu_{B}$ of the QCD phase diagram can be obtained from the momentum distributions and the ratios of the produced particles in heavy-ion collisions. Each collision energy corresponds to one $T$ - $\mu_{B}$ point in the phase diagram. So, idea is to collect data at different center-of-mass energies by colliding heavy-ions. Once, the $T-\mu_{B}$ point is obtained, one can look at the various signatures for the phase boundary, first-order phase transition, and the critical point. One of the interesting aspect is to locate the energy where the established signatures of the QGP (at top RHIC energy) disappear or "turn-off". This is how the RHIC beam energy scan program was planned 3445]. The proposal for the BES program was made in the year 2008. This was followed by a successful data taking and physics analysis of a test run of $\mathrm{Au}+\mathrm{Au}$ collisions below injection energies at $\sqrt{s_{N N}}=9.2$ $\mathrm{GeV} 11$. The first phase of the BES program was started in the year 2010 with data taking in $\mathrm{Au}+\mathrm{Au}$ collisions at three low energies of 7.7, 11.5, and $39 \mathrm{GeV}$. In 2011, two more energies at $\sqrt{s_{N N}}=19.6$ and $27 \mathrm{GeV}$ were included. Table 1 lists various 
energies and corresponding number of events collected by the STAR detector in 2010-2011 for Phase-I of the BES program.

Table 1. The data collected during the Phase-I of the BES program.

\begin{tabular}{c|c|c}
\hline Year & $\sqrt{s_{N N}}(\mathrm{GeV})$ & $N_{\text {event }}$ (Million) \\
\hline 2010 & 7.7 & 5 \\
2010 & 11.5 & 12 \\
2010 & 39 & 130 \\
2011 & 19.6 & 36 \\
2011 & 27 & 70 \\
\hline
\end{tabular}

This review is organized as follows. In Sec. 2, we discuss freeze-out parameters that provide information about $T-\mu_{B}$ points in the QCD phase diagram. In Sec. 3 , signatures of first-order phase transition and that for turn-off of QGP are discussed. These include results on freeze-out eccentricity, directed flow, elliptic flow, dynamical charge correlations, and nuclear modification factor. The signatures for the search of possible critical point are discussed in Sec. 4 that include energy dependence of particle ratio fluctuations and higher moments of conserved quantities such as net-proton. Section 5 provides the outlook for the BES Phase-II program. Finally, we conclude with a summary in Sec. 6.

\section{Freeze-out Parameters}

The QCD phase diagram is the variation of temperature $T$ and baryon chemical potential $\mu_{B}$. These quantities can be extracted from the measured hadron yields. Transverse momentum spectra for the BES Phase-I energies are obtained for $\pi, K$, $p, \Lambda, \Xi, K_{S}^{0}$, and $\phi \underline{12[13}$. From these distributions, corresponding particle yields are obtained and various particle ratios are constructed. These particle ratios are used to obtain the chemical freeze-out (a state after the collision when the yields of particles get fixed) conditions using the statistical thermal model (THERMUS) 14/15/16. The two main extracted parameters are chemical freeze-out temperature $T_{\mathrm{ch}}$ and $\mu_{B}$. Figure 2 (left panel) shows the variation of the extracted chemical freeze-out parameters using the Grand-Canonical Ensemble (GCE) approach of THERMUS for different energies and centralities 17/18. The curves represent the parameterizations of $T_{\mathrm{ch}}$ and $\mu_{B}$ 19|20. We observe that at top RHIC energy, there is a little variation of chemical freeze-out parameters with centrality. While at lower energies, $T_{\mathrm{ch}}$ shows a variation with $\mu_{B}$ as a function of centrality. The centrality dependence of these parameters is observed for the first time in heavy-ion collisions at these lower energies. One advantage of having such a dependence is that one can explore larger portion of the QCD phase diagram.

The particle spectra can be used to obtain the kinetic freeze-out (a state after the collision when the spectral shapes of particles get fixed) conditions using the Blast Wave (BW) model 21. The BW model is used to simultaneously fit the $\pi, K, p$ 

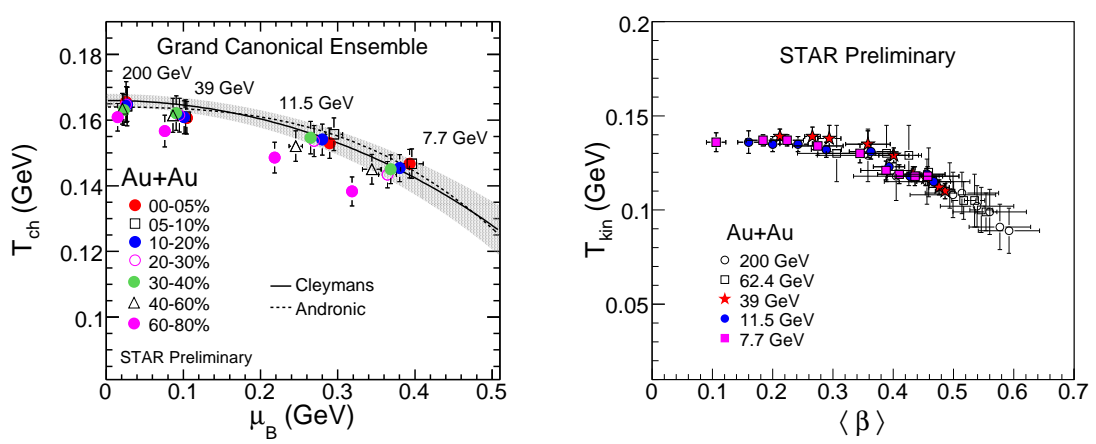

Fig. 2. (Color online) Left panel: Variation of $T_{\mathrm{ch}}$ with $\mu_{B}$ for different energies and centralities. Right panel: Variation of $T_{\text {kin }}$ with $\langle\beta\rangle$ for different energies and centralities. Errors in both panels represent the quadrature sum of systematic and statistical errors.

spectra and the two main extracted parameters are kinetic freeze-out temperature $T_{\text {kin }}$ and average flow velocity $\langle\beta\rangle$. Figure 2 (right panel) shows the variation of kinetic freeze-out parameters for different energies and centralities 18 . We observe that at a given collision energy, there is an anti-correlation between $T_{\text {kin }}$ and $\langle\beta\rangle$. For a given collision centrality, the freeze-out temperature at high energy is lower and the average collectivity velocity $\langle\beta\rangle$ is larger due to expansion.

\section{Search for First Order Phase Transition \& Turn-off of QGP Signatures}

Having discussed about accessing the QCD phase diagram by obtaining $T-\mu_{B}$ points, we can now discuss various signatures for first order phase transition or softest point in equation of state and those showing "turn-off" of QGP. These include freeze-out eccentricity, directed flow, elliptic flow, dynamical charge correlations, and nuclear modification factor.

\subsection{Freeze-out Eccentricity}

Eccentricity at freeze-out can be extracted as: $\epsilon_{F}=\frac{\sigma_{y}^{2}-\sigma_{x}^{2}}{\sigma_{y}^{2}+\sigma_{x}^{2}} \approx 2 R_{s, 2}^{2} / R_{s, 0}^{2}$, where $\sigma_{x}$ and $\sigma_{y}$ correspond to the widths of the participant zone at freeze-out in the in-plane and and out-of-plane directions, respectively $22 . R_{s, 2}^{2}$ and $R_{s, 0}^{2}$ are the $2^{n d_{-o r d e r} \text { and }}$ $0^{t h}$-order Fourier coefficients radius terms along the "side" direction (perpendicular to the direction of average transverse pair momentum or "out" and that along the beam direction or "long"), respectively. The ratio $R_{s, 2}^{2} / R_{s, 0}^{2}$ is less affected by flow so it carries mainly the geometric information. Freeze-out eccentricity may provide important information related to both the equation of state and dynamical processes involved in heavy-ion collisions as explained below. In non-central collisions, there is an initial anisotropy created (elliptic shape) that leads to more compres- 


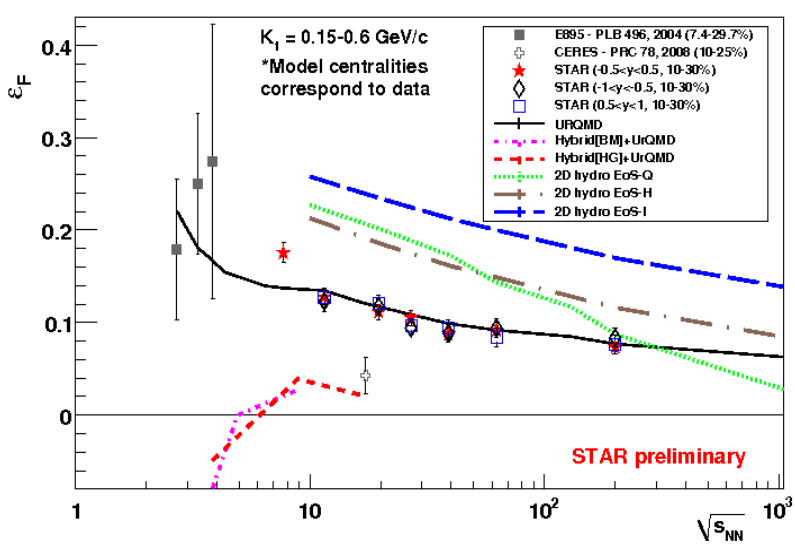

Fig. 3. (Color online) Freeze-out eccentricity as a function of beam energy compared to different model calculations.

sion along the shorter axis and hence larger initial pressure gradients. This might lead to expansion along the shorter axis thereby reducing the eccentricity. Ultimately, the system must evolve to a more round freeze-out shape. Increasing energy would lead to longer lifetimes and pressure gradients, and hence a monotonically decreasing excitation function for the freeze-out eccentricity would be expected. If the system undergoes a first-order phase transition, a mixed phase is expected. The system could spend more time in the mixed phase compared to that in other phases. This may lead to different expansions for different phases and hence non-monotonic freeze-out shape. Figure 3 shows the energy dependence of freeze-out eccentricity compared to several model calculations including UrQMD 23 and other 2D hydrodynamical models 24 . These results suggest a monotonic decrease in the freeze-out eccentricity with beam energy.

\subsection{Directed Flow}

The directed flow $v_{1}$ is calculated as $\left\langle\cos \left(\phi-\Psi_{1}\right)\right\rangle$, where $\phi$ and $\Psi_{1}$ are the azimuthal angle of the produced particles and orientation of the first-order event plane, respectively. The directed flow measurements near midrapidity for protons are proposed to be sensitive to the equation of state (EOS) 25/26/27. It has been predicted that proton $v_{1}$ slope show a non-monotonic behavior as a function of beam energy illustrating change of sign from positive to negative at lower energies and again going back to positive at higher energies 27. This is sometimes called collapse of proton flow. The minimum in proton $v_{1}$ slope is proposed to correspond to a softest point in equation of state. Figure 4 shows the results from the beam energy scan. Plotted here is the $v_{1}$ slope $\left(d v_{1} / d y^{\prime}\right.$, where $y^{\prime}=y / y_{\text {beam }}$ and $y$ is rapidity), near midrapidity as a function of beam energy for the mid-central (10-40\%) Au+Au collisions 28 . 


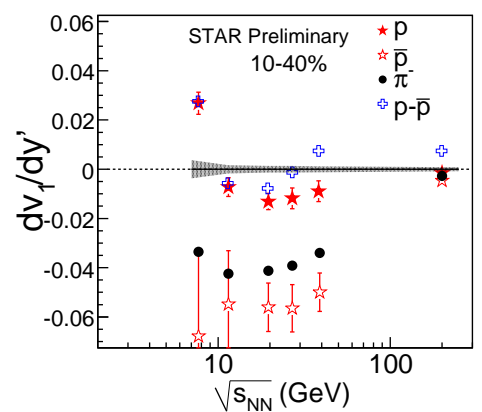

Fig. 4. (Color online) Directed flow slope $\left(d v_{1} / d y^{\prime}, y^{\prime}=y / y_{\text {beam }}\right)$ for $\pi^{-}, p, \bar{p}$, and netprotons $(p-\bar{p})$ near midrapidity as a function of beam energy for mid-central (10-40\%) $\mathrm{Au}+\mathrm{Au}$ collisions. The shaded band refers to the systematic uncertainty on net-proton measurements.

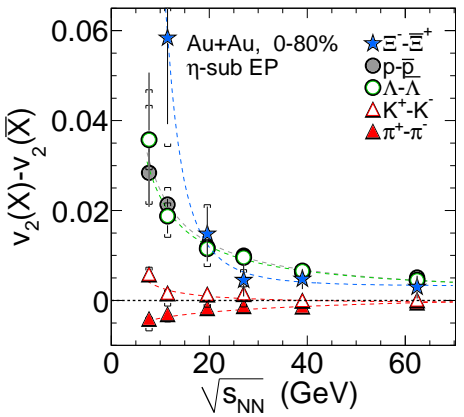

Fig. 5. (Color online) The difference in $v_{2}$ between particles and their corresponding anti-particles as a function of beam energy in $0-80 \% \mathrm{Au}+\mathrm{Au}$ collisions. The curves represent fits to data points as discussed in text. Both statistical (vertical lines) and systematic errors (caps) are shown.

The pion and anti-proton $v_{1}$ slopes show negative values for all the beam energies studied. The proton $v_{1}$ slope changes sign while going from $7.7 \mathrm{GeV}$ to $11.5 \mathrm{GeV}$ and then stays negative up to $200 \mathrm{GeV}$. However, the net-protons $v_{1}$ slope (obtained using $v_{1}$ slopes of $p, \bar{p}$ and ratio of $\bar{p} / p$ ) changes sign from positive to negative and again becomes positive as a function of beam energy. Both proton and net-proton $v_{1}$ slopes show a dip (or minimum) around $\sqrt{s_{N N}}=10-20 \mathrm{GeV}$. In order to quantify the minimum position, it will be interesting to add one more energy point around $15 \mathrm{GeV}$. Also more theoretical as well as experimental studies are needed in order to understand these interesting observations.

\subsection{Elliptic Flow}

The elliptic flow $v_{2}$ is calculated as $\left\langle\cos 2\left(\phi-\Psi_{2}\right)\right\rangle$, where $\Psi_{2}$ is orientation of the second-order event plane. Elliptic flow mainly probes the early stages of heavyion collisions. At top RHIC energy of $200 \mathrm{GeV}$ in $\mathrm{Au}+\mathrm{Au}$ collisions, the elliptic flow scaled by the number of constituent quarks $\left(n_{q}\right)$ versus $\left(m_{T}-m_{0}\right) / n_{q}$ (where $m_{T}=\sqrt{p_{T}^{2}+m_{0}^{2}}$ ) shows a scaling behavior where mesons and baryons have similar values at intermediate $p_{T}$. This is referred to as the number of constituent quark (NCQ) scaling 29. It is an established signature of partonic matter formed in $\mathrm{Au}+\mathrm{Au}$ collisions at $200 \mathrm{GeV}$ and deviations from such scaling would indicate the dominance of hadronic interactions. Hence breaking of NCQ scaling at lower energies could be an indication of a "turn-off" of QGP signatures. Figure 5 shows the difference in $v_{2}$ of particles and corresponding anti-particles as a function of beam energy 30. The curves represent fits to data points with functional form: $f_{\Delta v_{2}}\left(\sqrt{s_{N N}}\right)=a{\sqrt{s_{N N}}}^{-b}+c$. The $v_{2}$ difference between particles and anti-particles is observed to increase when we go towards the lower energies. At low energies, 


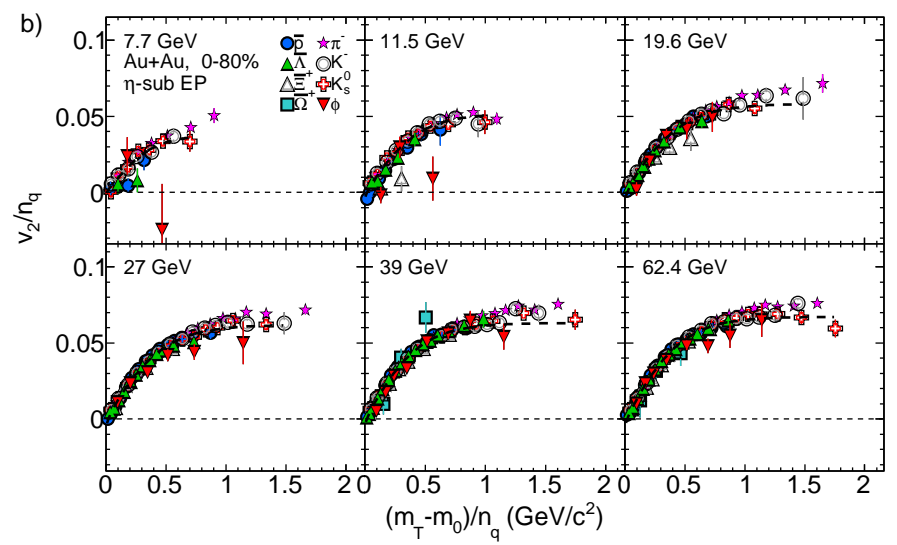

Fig. 6. (Color online) $v_{2} / n_{q}$ as a function of $\left(m_{T}-m_{0}\right) / n_{q}$ for different particles in $\mathrm{Au}+\mathrm{Au}$ collisions at $\sqrt{s_{N N}}=7.7,11.5,19.6,27,39$ and $62.4 \mathrm{GeV}$. The errors shown are statistical only.

$v_{2}\left(\pi^{-}\right)>v_{2}\left(\pi^{+}\right), v_{2}\left(K^{+}\right)>v_{2}\left(K^{-}\right)$, and $v_{2}$ (baryons) $>\mathrm{v}_{2}$ (anti-baryons). This difference between particles and anti-particles suggests that the NCQ scaling among particles and anti-particles is broken. However, the observed difference between $v_{2}$ of particles and anti-particles could be qualitatively explained by the models incorporating baryon transport at midrapidity and hadronic interactions $31 / 32$. We also observe that the baryons-mesons splitting for $v_{2}$ versus $m_{T}-m_{0}$ starts to disappear for anti-particles at $11.5 \mathrm{GeV}$ and below. Figure 6 shows the $v_{2} / n_{q}$ versus $\left(m_{T}-m_{0}\right) / n_{q}$ for different particles for $\sqrt{s_{N N}}=7.7-62.4 \mathrm{GeV}$ [30. We observe that results for all the particles are consistent among each other within $\pm 10 \%$ level, except for the $\phi$-mesons at 7.7 and $11.5 \mathrm{GeV}$. At the largest $m_{T}-m_{0}$ the $\phi$-meson data points deviate by $1.8 \sigma$ and $2.3 \sigma$ for $\sqrt{s_{N N}}=7.7$ and $11.5 \mathrm{GeV}$, respectively. Since $\phi$-mesons have smaller hadronic interaction cross-section, their smaller $v_{2}$ values could indicate that the hadronic interactions start to dominate over partonic effects for the systems formed at beam energies below $\sqrt{s_{N N}}=11.5 \mathrm{GeV} 33 \mid 34$. However, as can been seen from the figure, a higher statistics data are needed to extend the $m_{T}-m_{0}$ range and significance of the deviation observed.

\subsection{Dynamical Charge Correlations}

The dynamical charge correlations are studied through a three-particle mixed harmonics azimuthal correlator $35, \gamma=\left\langle\cos \left(\phi_{\alpha}+\phi_{\beta}-2 \Psi_{\mathrm{RP}}\right)\right\rangle$. This observable represents the difference between azimuthal correlations projected onto the direction of the angular momentum vector and correlations projected onto the collision reaction plane. It is suggested that the difference in the correlations between same sign and opposite sign charges in heavy-ion collisions could be related to local parity violation if there is a deconfinement and a chiral phase transition $\underline{36}$. This is also referred to 


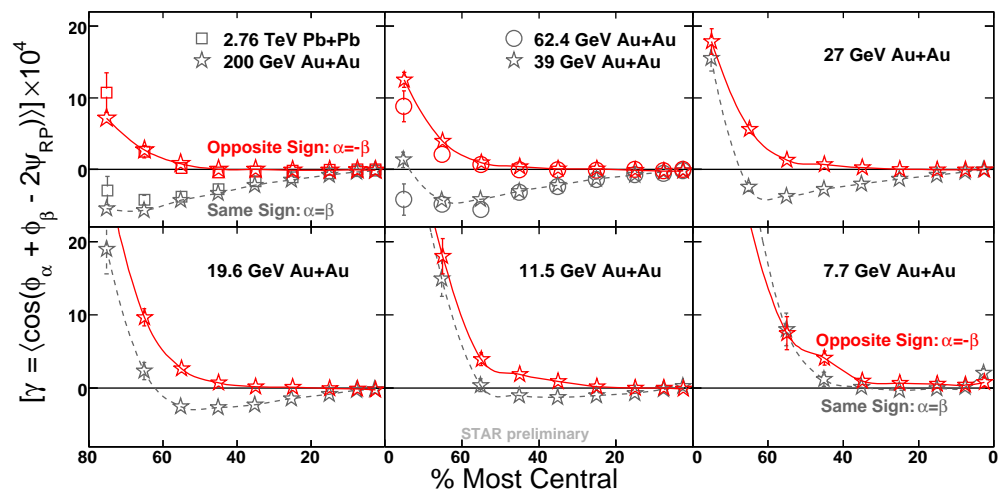

Fig. 7. (Color online) Dynamical charge correlations as a function of centrality for Au+Au collisions at $\sqrt{s_{N N}}=7.7-200 \mathrm{GeV}$. For comparison, results for $\mathrm{Pb}+\mathrm{Pb}$ collisions at $2.76 \mathrm{TeV}$ are also shown. Errors are statistical only.

as Chiral Magnetic Effect (CME). At top RHIC energies, we observed a separation between the correlations of same and opposite sign charges. If this difference can be attributed to the QCD phase transitions, the absence of such observation could be an indication of the system which did not undergo the phase transition. Hence, the observable could be useful to locate the energy in the BES program where the QGP signature "turns off". Figure 7 shows the results for the beam energies from $7.7-200 \mathrm{GeV}$ as a function of centrality 37 . For comparison, $\mathrm{Pb}+\mathrm{Pb}$ results from ALICE are also shown 38 which are observed to be consistent with the results from top RHIC energy. The separation between same and opposite sign charges decreases with decreasing energy and vanishes below $\sqrt{s_{N N}}=11.5 \mathrm{GeV}$.

\subsection{Nuclear Modification Factor}

Nuclear modification factor $R_{\mathrm{CP}}$ is one of the established observable for the signature of QGP at top RHIC energy [39]. It is defined as ratio of yields at central collisions to those at peripheral collisions, scaled by the corresponding number of binary collisions $N_{\text {bin }}$. The number of binary collisions are calculated from the Monte Carlo model. It has been observed that at high $p_{T}$, the $R_{\mathrm{CP}}$ of various particles is less than unity 39 , which is attributed to the energy loss of the partons in the dense medium. In the absence of dense medium, there may not be suppression of high $p_{T}$ particles, which can serve as an indication of "turn-off" of a QGP signature.

Figure 8 (left panel) shows the $R_{\mathrm{CP}}$ of $K_{S}^{0}$ in $\mathrm{Au}+\mathrm{Au}$ collisions at $\sqrt{s_{N N}}=7.7-$ $39 \mathrm{GeV}$ 40. We observe that for $p_{T}>2 \mathrm{GeV} / c$, the $R_{\mathrm{CP}}\left(K_{S}^{0}\right)$ is less than unity at $39 \mathrm{GeV}$ and then the value increases as the beam energy decreases. For $\sqrt{s_{N N}}<$ $19.6 \mathrm{GeV}, R_{\mathrm{CP}}\left(K_{S}^{0}\right)$ is above unity, indicating decreasing partonic effects at lower energies. Figure 8 (right panel) shows the $R_{\mathrm{CP}}$ results for charged hadrons in $\mathrm{Au}+\mathrm{Au}$ collisions at $\sqrt{s_{N N}}=7.7-200 \mathrm{GeV}$ 41. Again, we observe no suppression at lower 

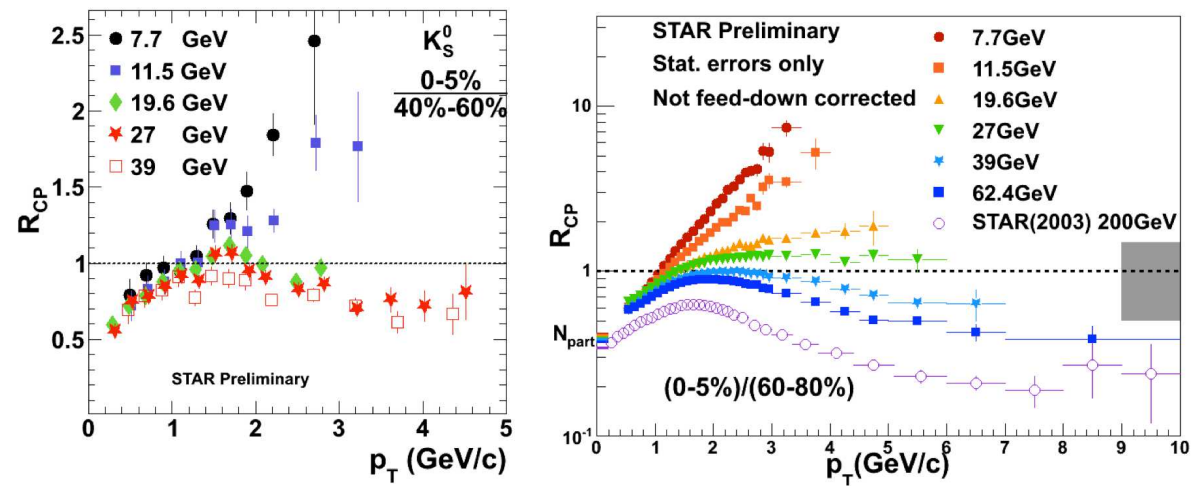

Fig. 8. (Color online) Left: $R_{\mathrm{CP}}\left(\frac{0-5 \%}{40 \%-60 \%}\right)$ for $K_{S}^{0}$ in $\mathrm{Au}+\mathrm{Au}$ collisions at $\sqrt{s_{N N}}=7.7-39 \mathrm{GeV}$. Errors are statistical only. Right: $R_{\mathrm{CP}}\left(\frac{0-5 \%}{60 \%-80 \%}\right)$ for charged hadrons in $\mathrm{Au}+\mathrm{Au}$ collisions at $\sqrt{s_{N N}}=7.7-200 \mathrm{GeV}$. Grey band corresponds to the systematic uncertainty.

energies for $p_{T}>2 \mathrm{GeV} / c$, supporting the $R_{\mathrm{CP}}\left(K_{S}^{0}\right)$ results. Both results suggest that partonic effects become less important at lower energies and the cold nuclear matter effects (Cronin effect) start to dominate at these energies $\underline{42}$.

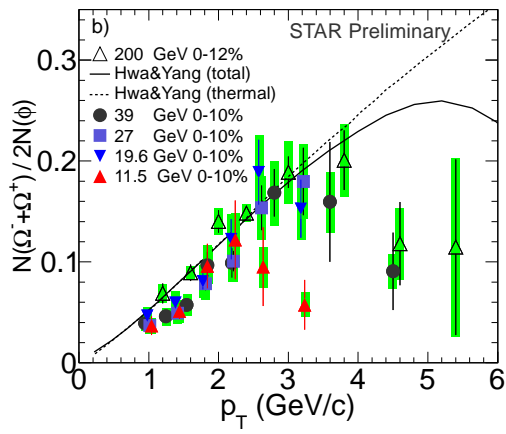

Fig. 9. (Color online) The baryon to meson ratio $N\left(\Omega^{-}+\Omega^{+}\right) /(2 N \phi)$ as a function of $p_{T}$ in central $\mathrm{Au}+\mathrm{Au}$ collisions at $\sqrt{s_{N N}}=11.5-200 \mathrm{GeV}$. The curves represent model calculations by Hwa and Yang for $\sqrt{s_{N N}}=200 \mathrm{GeV}$. Both statistical errors (vertical lines) and systematic errors (shaded bands) are shown.

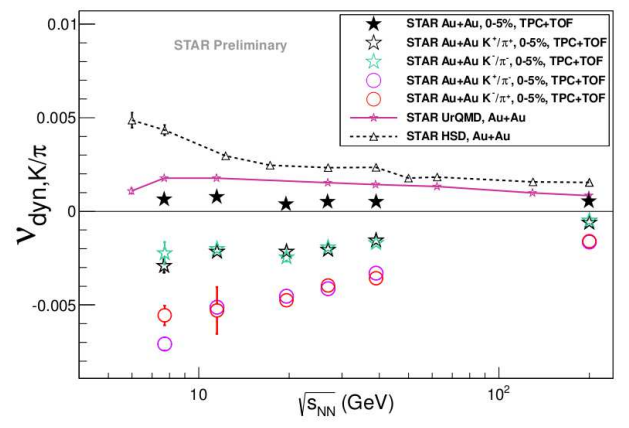

Fig. 10. (Color online) $\nu_{\mathrm{dyn}}$ for $K / \pi$ (along with $K^{+} / \pi^{+}, K^{-} / \pi^{-}, K^{+} / \pi^{-}$, and $K^{-} / \pi^{+}$) ratio in $0-5 \%$ central $\mathrm{Au}+\mathrm{Au}$ collisions are shown as a function of energy. Results are compared with transport models such as UrQMD and HSD. Errors are statistical.

Figure 9 shows the baryon to meson ratio $N\left(\Omega^{-}+\Omega^{+}\right) /(2 N \phi)$ as a function of $p_{T}$ in central $\mathrm{Au}+\mathrm{Au}$ collisions at $\sqrt{s_{N N}}=11.5-200 \mathrm{GeV}$. The curves represent model calculations by Hwa and Yang in central collisions at $\sqrt{s_{N N}}=200 \mathrm{GeV} 43 \mid 44$ which assume the $\Omega$ and $\phi$ yields to be generated from the recombination of thermal strange quarks having exponential $p_{T}$ distribution. The particle ratio results at 
$\sqrt{s_{N N}}=19.6,27$, and $39 \mathrm{GeV}$ seem to follow that of $200 \mathrm{GeV}$, indicating a maximum around $p_{T} \geq 3 \mathrm{GeV} / c$ and then turning down as the $p_{T}$ is increased. However, results at $11.5 \mathrm{GeV}$ show different behavior i.e. show a maximum at somewhat lower $p_{T}$ of $\sim 2 \mathrm{GeV} / c$ before turning down for higher values of $p_{T}$. This observation suggests that there might be a significant change in the underlying $p_{T}$ distributions of strange quarks recombining to form final $\Omega$ and $\phi$ for $\sqrt{s_{N N}}=11.5 \mathrm{GeV}$ and those for $\sqrt{s_{N N}} \geq 19.6 \mathrm{GeV}$.

\section{Search for QCD Critical Point}

In this section, we discuss the observables that could possibly be related to the critical point search. First, we discuss the particle ratio fluctuations such as $K / \pi$ ratio fluctuations as a function of beam energy. After that, we discuss about the conserved number fluctuations that include net-proton higher moments results.

\section{1. $K / \pi$ Ratio Fluctuations}

If a system passes close to a critical point, large density variations or enhanced fluctuations are expected e.g. as seen in critical opalescence. From experimental side, one expects a non-monotonic variation of a potential observable as a function of beam energy. Dynamical particle ratio fluctuations such as $K / \pi, p / \pi$, and $K / p$, might be sensitive to the initial state fluctuations arising from the existence of critical point 45/46. The observable used to quantify these (e.g. $K / \pi$ ) dynamical fluctuations $\nu_{\mathrm{dyn}}$ is given by

$$
\nu_{d y n, K / \pi}=\frac{\left\langle N_{K}\left(N_{K}-1\right)\right\rangle}{\left\langle N_{K}\right\rangle^{2}}+\frac{\left\langle N_{\pi}\left(N_{\pi}-1\right)\right\rangle}{\left\langle N_{\pi}\right\rangle^{2}}-2 \frac{\left\langle N_{K} N_{\pi}\right\rangle}{\left\langle N_{K}\right\rangle\left\langle N_{\pi}\right\rangle},
$$

where $N_{K}$ and $N_{\pi}$ are the average number of kaons and pions in an event, respectively. For a pure Poisson distribution, $\nu_{d y n, K / \pi}$ will be zero. Figure 10 shows the $\nu_{\text {dyn }}$ results for $K / \pi$ (along with $K^{+} / \pi^{+}, K^{-} / \pi^{-}, K^{+} / \pi^{-}$, and $K^{-} / \pi^{+}$) ratio in $0-5 \%$ central collisions as a function of beam energy 47 . The dynamical $K / \pi$ ratio fluctuations show a smooth or monotonic behavior as a function of beam energy. The transport models such as HSD 48 and UrQMD 23 show a similar smooth dependence on beam energy as observed in data.

\subsection{Conserved Number Fluctuations}

Higher moments of conserved number fluctuations are proposed to be potential observables for the search of the critical point 49|50|51. For a static, infinite medium, the correlation length $\xi$ diverges at critical point. The various moments of event-byevent conserved numbers (such as net-baryons, net-charge, and net-strangeness) distributions are related to different powers of the correlation length. Higher moments such as skewness $S$ and kurtosis $\kappa$ are related to higher power of the correlation length 52153 . Thus, these higher moments have a better sensitivity for the search of 
the critical point. It has been proposed that the appropriate products of these moments such as $\kappa \sigma^{2}$ and $S \sigma$ can be related to the ratios of order susceptibilities calculated in lattice QCD and HRG model as $\kappa \sigma^{2}=\chi_{B}^{(4)} / \chi_{B}^{(2)}$ and $S \sigma=\chi_{B}^{(3)} / \chi_{B}^{(2)}$ 54/55. One of the advantages of using these products or ratios is that they cancel the volume effects which are difficult to estimate in an experiment. So in this way, one can relate the experimental measurements with the lattice QCD observables for the search of critical point. Since in an experiment, it is difficult to obtain total baryons on an event-by-event basis, net-protons are used as a proxy for the net-baryons.

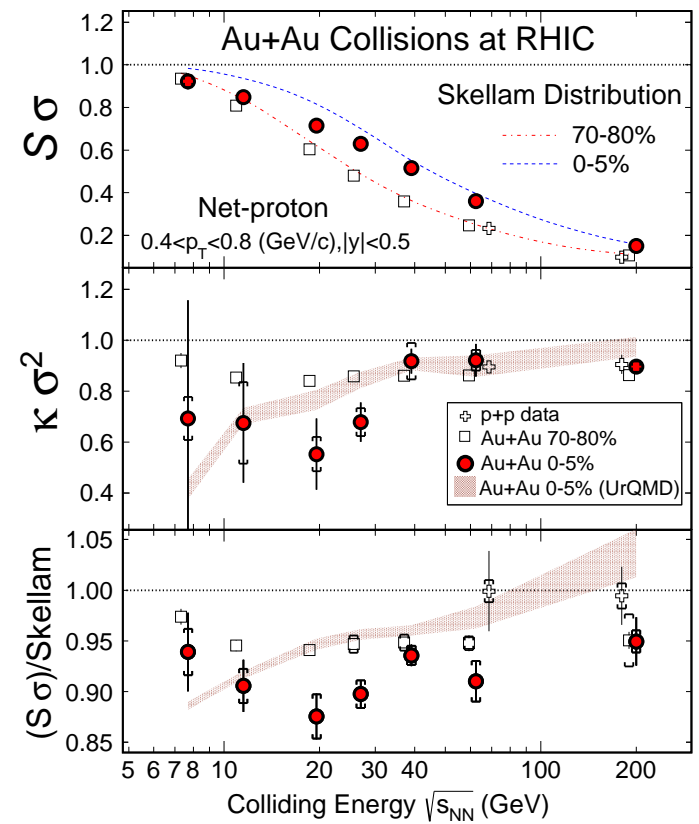

Fig. 11. (Color online) $\kappa \sigma^{2}, S \sigma$ and $S \sigma$ values normalized by the Skellam expectations as a function of collision energy and two different centralities. Results from $\mathrm{p}+\mathrm{p}$ collisions are also shown. All the results presented are corrected for detector efficiency. Results from UrQMD model calculations are also shown. The widths of bands represent statistical uncertainties. The error bars on data points are statistical while caps represent the systematic errors.
Figure 11 shows the $\kappa \sigma^{2}$ and $S \sigma$ for net-protons as a function of beam energy for different collision centralities 56/57. For comparison, the results are shown for Skellam expectations and UrQMD model calculations that do not include critical point 23 . The results from $p+p$ collisions at $200 \mathrm{GeV}$ are also shown. The bottom panel shows the $S \sigma$ values normalized by the corresponding Skellam expectations. We observe that the moment products $\kappa \sigma^{2}$ and $S \sigma$ show similar values for central for central $0-5 \%$ and peripheral collisions $(70-80 \%)$ for $\sqrt{s_{N N}}=39$ $200 \mathrm{GeV}$. For beam energies below $39 \mathrm{GeV}$, they have different values for central and peripheral collisions. These values are below Skellam expectations for $\sqrt{s_{N N}}>7.7 \mathrm{GeV}$ for $0-5 \%$ central collisions. The deviation from Skellam expectation is observed to be more significant at $\sqrt{s_{N N}}=19.6$ and $27 \mathrm{GeV}$. The UrQMD model calculations show

a smooth monotonic behavior as a function of collision energy. There are large uncertainties for data points below $19.6 \mathrm{GeV}$ that call for higher statistics data at these energies. In addition, a direct comparison to QCD calculations with critical point obtained using similar dynamics at that of heavy-ion collisions can provide definite answer about the existence of critical point. 


\section{BES Phase-II}

The first phase of the BES program has yielded several promising results for the understanding of QCD phase diagram. Some of the observables require high statistics data to make definite statements. These include $\phi$-meson $v_{2}$ to test the NCQ scaling hypothesis at lower energies and higher moments of net-protons to see whether there is a non-monotonic variation towards lower energies that could suggest a possible critical point. In addition, energy dependence of some observables suggest

Table 2. Proposed energies, $\mu_{B}$ values, and required number of events for the BES Phase-II. Also listed are the corresponding fixed target $\sqrt{s_{N N}}$, centre of mass rapidity, and $\mu_{B}$ reach.

\begin{tabular}{c|c|c|c|c|c}
\hline \multicolumn{3}{c|}{ BES Phase - II } & \multicolumn{3}{c}{ Fixed Target Collisions } \\
\hline$\sqrt{s_{N N}}(\mathrm{GeV})$ & $\mu_{B}(\mathrm{MeV})$ & $N_{\text {event }}$ (Million) & $\sqrt{s_{N N}}(\mathrm{GeV})$ & $y_{\mathrm{CM}}$ & $\mu_{B}(\mathrm{MeV})$ \\
\hline 19.6 & 205 & 400 & 4.5 & 1.52 & 585 \\
15 & 250 & 100 & 4.0 & 1.39 & 620 \\
11.5 & 315 & 120 & 3.5 & 1.25 & 670 \\
7.7 & 420 & 80 & 3.0 & 1.05 & 720 \\
\hline
\end{tabular}

to have a need of one more energy point around $15 \mathrm{GeV}$. For example, proton and net-proton $v_{1}$ slopes suggest a minimum around $11.5-19.6 \mathrm{GeV}$ as a function of energy which could be related to the softest point in equation of state. Having one more energy point in between would indicate the exact location of the minimum. Similar reason (although there is a monotonic variation as a function of beam energy at the moment) might be argued for the freeze-out eccentricity. For net-proton higher moments, adding $15 \mathrm{GeV}$ along with high-statistics data at lower energies might provide the clear energy dependence trend with high significance. One more energy point at 15 $\mathrm{GeV}$ is also important in view of the fact that the gap be-

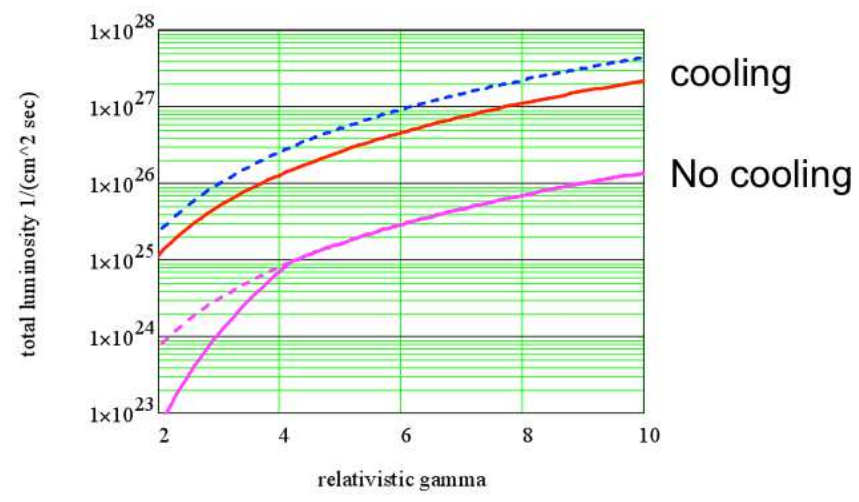

Fig. 12. (Color online) Improvement in RHIC luminosity for the lower energies with electron cooling and long bunches (with space charge tune spread $\Delta Q_{\mathrm{SC}}=0.05$ and $\sigma_{s}=3 \mathrm{~m}$.)

tween 11.5 and $19.6 \mathrm{GeV}$ in terms of $\mu_{B}$ is more than $100 \mathrm{MeV}$.

For the reasons mentioned above, RHIC has decided to continue the exploration of QCD phase diagram and hence proposed a second phase of the BES program. The proposal for BES Phase-II includes high statistics data below $20 \mathrm{GeV}$ as listed in 
the Table 2, To achieve the high statistics data at lower energies, an electron cooling device is requested to be installed at RHIC for increasing the beam luminosity 58 . Simulation results (see Fig. 12) indicate that with electron cooling, a significant improvement can be made to increase luminosity (as shown by red solid curve in the figure). An additional improvement in luminosity (as shown by blue dashed curve) may be possible by operating with longer bunches at the space-charge limit in the collider 59. Electron cooling may increase the luminosity by a factor of $3-$ 10 and with longer bunches the luminosity may be increased by another factor of 2-5. The high statistics data from BES Phase-II will not only allow the precision measurements of the important observables discussed here but will also be helpful in the measurements of rare probes such as dilepton production and hypertriton measurements at lower energies 60161.

To maximize the use of collisions provided at STAR, a fixed target proposal is made along with BES Phase-II. The idea is to install a fixed Au target inside the STAR beam pipe to perform the $\mathrm{Au}$ (beam)-Au(target) collisions. Such collisions will provide lower reach for the center of mass energies and higher reach for the $\mu_{B}$ values for a given BES Phase-II energy. The beam energies and the $\mu_{B}$ values for the fixed target collisions are listed in the Table 2 corresponding to the proposed BES Phase-II energies. The $\mu_{B}$ values are obtained from the parameterizations in Ref. 20. Clearly, it provides an opportunity to reach the large values of $\mu_{B}$ and hence to explore the larger portion of the QCD phase diagram. One of the advantages for such a proposal is that the data taking for these fixed target collisions can be done concurrently during the normal RHIC running and hence it will not affect the normal RHIC operations.

These programs will also benefit from the proposed inner sector upgrade of STAR TPC called the iTPC upgrade 62 . At the moment, inner sector of the TPC has the following issues: the inner sector wires are showing the signs of ageing and unlike the outer TPC sectors, it does not have the hermetic coverage at all radii. The spacing between the rows is greater than $5 \mathrm{~cm}$ which results in missing rows. To overcome these issues, it has been proposed to increase the segmentation on the inner pad plane and renew the inner sector wires. Simulation studies suggest that with iTPC upgrade it is possible obtain better momentum resolution, better $d E / d x$ resolution for particle identification, and improved acceptance at higher pseudorapidity $\eta$ and low $p_{T}$. At the moment, TPC $\eta$ coverage is about $|\eta|<1.0$, however, with iTPC upgrade, it might reach $|\eta|<1$.7. Similarly, lowest $p_{T}$ achieved can be as low as 60 $\mathrm{MeV} / c$ compared to the present value of $125 \mathrm{MeV} / c$. The above listed improvements will definitely strengthen the technical aspects in the Physics analyses proposed for the BES Phase-II. The BES Phase-II is expected to start around 2018-2019.

\section{Summary}

The BES Phase-I enables RHIC to cover large range of $\mu_{B}(20-400 \mathrm{MeV})$ in the phase diagram. At lower energies, a centrality dependence of freeze-out parameters 
is observed. The observables such elliptic flow $v_{2}$, nuclear modification factor $R_{\mathrm{CP}}$, baryon to meson ratio $\Omega / \phi$, dynamical charge correlations, suggest that hadronic interactions dominate for $\sqrt{s_{N N}} \leq 11.5 \mathrm{GeV}$ or that the system did not undergo QGP phase transition at these lower energies. The (net)-proton directed flow $v_{1}$ slope show interesting behavior for the energy range $\sqrt{s_{N N}}<20 \mathrm{GeV}$. The proton $v_{1}$ slope changes sign between 7.7 and $11.5 \mathrm{GeV}$. The net-proton $v_{1}$ slope changes sign twice as a function of beam energy. Both proton and net- proton $v_{1}$ slopes show a minimum around $11.5-19.6 \mathrm{GeV}$. The $\kappa \sigma^{2}$ and $S \sigma$ for net-protons show most significant deviations from Skellam expectations at $\sqrt{s_{N N}}=19.6$ and 27 $\mathrm{GeV}$. BES Phase-II along with electron cooling, fixed target proposal, and iTPC upgrade provides optimistic future for the exploration of the QCD phase diagram, and hence for critical point and phase boundary search.

We thank Prof. D. Keane, Prof. B. Mohanty, and Prof. Nu Xu for reading the manuscript and providing helpful comments and suggestions.

\section{References}

1. J. Adams et al. (STAR Collaboration), Nucl. Phys. A 757, 28 (2005).

2. V. Roy, A. K. Chaudhuri and B. Mohanty, Phys. Rev. C 86, 014902 (2012).

3. M. M. Aggarwal et al. (STAR Collaboration), arXiv:1007.2613.

4. L. Kumar (for STAR Collaboration), Nucl. Phys. A 862, 125 (2011);

5. B. Mohanty, Nucl. Phys. A 830, 899C (2009).

6. USA-NSAC 2007, Long range plan.

7. Y. Aoki, G. Endroli, Z. Fodor, S. D. Katz and K. K. Szabo, Nature 443 675, (2006).

8. S. Ejiri, Phys. Rev. D 78, 074507 (2008).

9. E. S. Bowman and J. I. Kapusta, Phys. Rev. C 79, 015202 (2009).

10. M. A. Stephanov, Prog. Theor. Phys. Suppl. 153, 139 (2004) [Int. J. Mod. Phys. A 20, 4387 (2005)] hep-ph/0402115.

11. B. I. Abelev et al. (STAR Collaboration), Phys. Rev. C 81, 024911 (2010).

12. L. Kumar (for STAR Collaboration), J. Phys. G: Nucl. Part. Phys. 38, 124145 (2011);

13. X. Zhu (for STAR Collaboration), Acta Phys. Polon. B Proc. Supp. 5, 213 (2012).

14. J. Adams et al. (STAR Collaboration), Nucl. Phys. A 757, 102 (2005);

15. A. Andronic, F. Beutler, P. Braun-Munzinger, K. Redlich and J. Stachel, Phys. Lett. B 675, 312 (2009) arXiv:0804.4132 [hep-ph]].

16. S. Wheaton and J. Cleymans, Comput. Phys. Commun. 180, 84 (2009) hep-ph/0407174.

17. L. Kumar [STAR Collaboration], Nucl. Phys. A 904-905, no. issue, 256c (2013) arXiv:1211.1350 [nucl-ex]].

18. S. Das [STAR Collaboration], Nucl. Phys. A 904-905, 891c (2013) arXiv:1210.6099 [nucl-ex]].

19. A. Andronic, P. Braun-Munzinger and J. Stachel, Nucl. Phys. A 834, 237C (2010) arXiv:0911.4931 [nucl-th]].

20. J. Cleymans, H. Oeschler, K. Redlich and S. Wheaton, Phys. Rev. C 73, 034905 (2006) hep-ph/0511094.

21. E. Schnedermann, J. Sollfrank and U. W. Heinz, Phys. Rev. C 48, 2462 (1993) nucl-th/9307020.

22. E. Mount, G. Graef, M. Mitrovski, M. Bleicher and M. A. Lisa, Phys. Rev. C 84, 014908 (2011) arXiv:1012.5941 [nucl-th]]. 
23. M. Bleicher, E. Zabrodin, C. Spieles, S. A. Bass, C. Ernst, S. Soff, L. Bravina and M. Belkacem et al., J. Phys. G 25, 1859 (1999) hep-ph/9909407.

24. M. A. Lisa, E. Frodermann, G. Graef, M. Mitrovski, E. Mount, H. Petersen and M. Bleicher, New J. Phys. 13, 065006 (2011) [arXiv:1104.5267 [nucl-th]].

25. J. Brachmann, S. Soff, A. Dumitru, H. Stoecker, J. A. Maruhn, W. Greiner, L. V. Bravina and D. H. Rischke, Phys. Rev. C 61, 024909 (2000) nucl-th/9908010.

26. L. P. Csernai and D. Rohrich, Phys. Lett. B 458, 454 (1999) nucl-th/9908034].

27. H. Stoecker, Nucl. Phys. A 750, 121 (2005) nucl-th/0406018.

28. Y. Pandit [STAR Collaboration], Nucl. Phys. A 904-905, 357c (2013). arXiv:1210.5315 [nucl-ex]].

29. J. Adams et al. (STAR Collaboration), Phys. Rev. Lett. 95, 122301 (2005).

30. L. Adamczyk et al. (STAR Collaboration), Phys. Rev. Lett. 110, 0142301 (2013).

31. J. C. Dunlop, M. A. Lisa and P. Sorensen, Phys. Rev. C 84, 044914 (2011) arXiv:1107.3078 [hep-ph]].

32. J. Xu, L. -W. Chen, C. M. Ko and Z. -W. Lin, Phys. Rev. C 85, 041901 (2012) arXiv:1201.3391 [nucl-th]].

33. B. Mohanty and N. Xu, J. Phys. G 36, 064022 (2009).

34. M. Nasim, B. Mohanty and N. Xu, Phys. Rev. C 87, 014903 (2013).

35. B. I. Abelev et al. (STAR Collaboration), Phys. Rev. Lett. 103, 251601 (2009).

36. K. Fukushima, D. E. Kharzeev and H. J. Warringa, Phys. Rev. D 78, 074033 (2008) arXiv:0808.3382 [hep-ph]].

37. G. Wang (for STAR Collaboration), Nucl. Phys. A 904-905, 248c (2013).

38. B. Abelev et al. [ALICE Collaboration], Phys. Rev. Lett. 110, 012301 (2013) arXiv:1207.0900 [nucl-ex]].

39. M. A. C. Lamont (for the STAR Collaboration), J. Phys. Conf. Ser. 50, 192 (2006).

40. X. Zhang (for STAR Collaboration), Nucl. Phys. A 904-905, 543c (2013).

41. E. Sangaline (for STAR Collaboration), Nucl. Phys. A 904-905, 771c (2013).

42. J. W. Cronin, H. J. Frisch, M. J. Shochet, J. P. Boymond, R. Mermod, P. A. Piroue and R. L. Sumner, Phys. Rev. D 11 (1975) 3105.

43. R. C. Hwa and C. B. Yang, Phys. Rev. C 75, 054904 (2007) nucl-th/0602024.

44. R. C. Hwa and C. B. Yang, Phys. Rev. C 66, 025205 (2002) hep-ph/0204289.

45. C. Pruneau, S. Gavin, and S. Voloshin, Phys. Rev. C 66, 044904 (2002).

46. J. Adams et al. (STAR Collaboration), Phys. Rev. C 68, 044905 (2003).

47. P. Tribedy (for STAR Collaboration), Nucl. Phys. A 904-905, 463c (2013).

48. M. I. Gorenstein, M. Hauer, V. P. Konchakovski and E. L. Bratkovskaya, Phys. Rev. C 79, 024907 (2009) arXiv:0811.3089 [nucl-th]].

49. M. M. Aggarwal et al. (STAR Collaboration), Phys. Rev. Lett. 105, 022302 (2010) arXiv:1004.4959 [nucl-ex]].

50. S. Gupta, X. Luo, B. Mohanty, H. G. Ritter and N. Xu, Science 332, 1525 (2011) arXiv:1105.3934 [hep-ph]].

51. F. Karsch and K. Redlich, Phys. Lett. B 695, 136 (2011).

52. M. A. Stephanov, Phys. Rev. Lett. 102, 032301 (2009);

53. M. A. Stephanov, Phys. Rev. Lett. 107, 052301 (2011).

54. R. V. Gavai and S. Gupta, Phys. Lett. B 696, 459 (2011) arXiv:1001.3796 [hep-lat]].

55. M. Cheng, P. Hendge, C. Jung, F. Karsch, O. Kaczmarek, E. Laermann, R. D. Mawhinney and C. Miao et al., Phys. Rev. D 79, 074505 (2009) arXiv:0811.1006 [hep-lat]].

56. X. Luo (for STAR Collaboration), Nucl. Phys. A 904-905, 911c (2013).

57. L. Adamczyk et al. (STAR Collaboration), arXiv:1309.5681.

58. A. Fedotov, and W. Fischer, Private communications, 2012.

59. A. Fedotov and M. Blaskiewicz, BNL CAD Tech Note: C-A/AP/449 (February 10, 
2012).

60. B. Huang (for STAR Collaboration), Nucl. Phys. A 904-905, 565c (2013).

61. Y. Zhu (for STAR Collaboration), Nucl. Phys. A 904-905, 551c (2013).

62. Y. Xu (for STAR Collaboration), poster "Inner TPC Upgrade at STAR", 2013 RHIC \& AGS Annual Users Meeting, BNL, USA. 